\title{
Fresnel vs. Einstein - a New Direct Test
}

\author{
Dan Wagner ${ }^{1}$ \\ ${ }^{1} 15$ Bradley Road, Madison, CT 06443, USA \\ Correspondence: Dan Wagner, 15 Bradley Road, Madison, CT 06443, USA. E-mail: drwagner10@comcast.net
}

Received: February 21, $2015 \quad$ Accepted: April 12, $2015 \quad$ Online Published: April 15, 2015
doi:10.5539/apr.v7n3p33
URL: http://dx.doi.org/10.5539/apr.v7n3p33

\begin{abstract}
When the phase velocity of light in a moving medium is used to predict the phase velocity of that light relative to a stationary observer then the Einstein low speed approximation to his velocity addition equation and the Fresnel drag equation both predict the exact same result. Therefore existing interference fringe shift experiments do not differentiate between the Fresnel and Einstein predictions. However, when the group velocity of light is used, the Fresnel prediction and the Einstein prediction are different. When Fresnel predictions based on group velocity are made for the proposed experiment, the difference in round trip arrival times for the two halves of a split laser pulse is substantial. When Einstein predictions based on group velocity are made the difference in round trip times is, of course, zero. Thus a positive result for the proposed experiment would violate Special Relativity Theory (SRT) with regards to the relativity of simultaneity and suggest that the Lorentz-Poincare' physical viewpoint on the SRT equations is a better viewpoint.

If the Einstein prediction for group velocity is not correct, then when positive data for the experiment configuration shown in Figure 1.0 is collected at various orientations at different times of the day it is possible to identify a local preferred reference frame in which the speed of light is actually (not just apparently) isotropic. This reference frame could be used in place of star reference frames for spacecraft navigation. Other implications of a local preferred reference frame are explained in the paper.
\end{abstract}

Keywords: phase velocity of light, group velocity of light, special relativity theory, Fresnel drag theory, relativity of simultaneity, Lorentz-Poincare' physical viewpoint, preferred reference frame, Einstein velocity addition formula, isotropic, anisotropy, CMBR, oscilloscope, pulsed laser, fiber optic, M. Hoek experiment

\section{Introduction}

In a paper by Wagner (2008, May/June) and in a subsequent paper by Wagner (2011, July 6-9), an experiment was proposed to decide whether Fresnel's or Einstein's equations more accurately combine moving medium velocity with group light velocity in the medium to obtain group light velocity relative to a stationary observer. Neither Fresnel nor Einstein use simple velocity addition.

Since the publications of these papers it has become apparent that a simple key point buried in the earlier, more detailed, paper is somewhat lost to the reader because the paper notations differ from Einstein's notions and even though their relationship is specified, the reader must put in an unnecessary amount of effort to gain understanding. Once this point is understood, there is ample reason to believe that the experiment proposed in the earlier papers could produce results which differ from those predicted by Special Relativity Theory (SRT). This brief paper is intended to clarify the point and thereby generate support within the scientific community for actually performing the proposed experiment.

\section{Einstein Velocity Addition Low Speed Approximation}

In a book by Einstein (1952), he says that for velocities that are small compared to the velocity of light, the SRT velocity-addition formula gives an approximation that is verified by the Fizeau experiment (i.e. the approximation matches the Fresnel drag equation).

In Einstein's notation, the SRT velocity addition equation is given by;

$$
W=\frac{v+w}{1+v w / c^{2}}
$$


where $v$ is the speed of the medium relative to the stationary observer, as measured by the stationary observer, $w$ is the speed of light in the medium relative to the medium, as measured by an observer moving with the medium, $W$ is the speed of light in the medium relative to the stationary observer, as measured by the stationary observer, and $\mathrm{c}$ is the speed of light in a vacuum.

In a footnote at the bottom of page 40, Einstein gives the following equation as a low speed approximation to his velocity addition equation.

$$
W=(w+v)\left(1-v w / c^{2}\right)
$$

Evidently he then makes an additional same order approximation resulting in the following final low velocity approximation to his velocity addition equation.

$$
W=w+v\left(1-w^{2} / c^{2}\right)
$$

If $\mathrm{n}$ is the refractive index of the transparent medium then $w=c / n$ and it follows that $1 / n^{2}=w^{2} / c^{2}$.

Substituting into the above equation we have;

$$
W=c / n+v\left(1-1 / n^{2}\right)
$$

Which is exactly the same as the Fresnel drag equation.

In commenting on the Fizeau experiment Einstein says, "In accordance with the principle of relativity we shall certainly have to take for granted that the propagation of light always takes place with the same velocity $w$ with respect to the liquid, whether the latter is in motion with reference to other bodies or not". The velocity referred to here is the phase velocity of light and its magnitude is $w=c / n$. In other words, this must be the phase velocity of light measured by someone moving with the liquid because otherwise he could detect his motion relative to an unknown preferred reference frame, where the speed of light is the same in all directions, and thereby violate the principle of relativity.

In addition to the phase velocity of light in a transparent medium there is also a very important 'group velocity' of light, which in general is quite a bit less than the phase velocity. The group velocity is important because it specifies how fast energy, and thus information, is transferred. It is the group velocity that is measured in 'direct' experiments where the light path and time of travel are measured directly as in the experiment proposed in the earlier papers. The group velocity of light in a stationary medium is a function of the medium and the wavelength of light used. For a specified medium and wavelength it has a constant magnitude which may be expressed as the phase magnitude minus a constant $G$;

$$
w=c / n-G
$$

$G$ is given by, $G=\lambda \frac{d(c / n)}{d \lambda}$, where $\lambda$ is the wavelength of the light used. This equation can also be written as $G=\lambda c \frac{d(1 / n)}{d \lambda}$. The value of $G$ for synthetic fused silica at $20 \mathrm{deg}$ C, light wavelength of $587.6 \mathrm{~nm}$, and refractive index of 1.4585 was determined from a table giving values for the refractive index of synthetic fused silica as a function of wavelength. The value determined is $G=2,960,760 \mathrm{~m} / \mathrm{s}$.

Einstein's reasoning must also apply to group velocity because for Special Relativity Theory this is simply another velocity in the moving medium and again if it were different from the stationary case it could be used to detect the medium's motion with respect to a preferred reference frame. Thus it appears correct to use equation (5) as the group speed of light in a moving medium relative to the medium, as measured by someone moving with the medium. We can therefore substitute equation (5) into equation (3) to obtain the Einstein low velocity approximation of the group velocity of light, $W$, relative to the stationary observer, as measured by the stationary observer.

$$
W=c / n-G+v\left(1-\frac{(c / n-G)^{2}}{c^{2}}\right)
$$

\section{Fresnel Velocity Addition (Drag) Equation}

For Fresnel, the phase velocity of light is considered to be the same in all directions, with a magnitude of $c / n$, in the preferred reference frame which is partially dragged by a moving medium. In other words light in the dragged reference frame is considered to propagate relative to the dragged reference frame with the same velocity as it propagates relative to the medium when the medium is stationary. Fresnel considers the preferred 
reference frame to be partially dragged at a speed of $v\left(1-1 / n^{2}\right)$, where $v$ is the medium speed relative to the preferred reference frame. Therefore, the Fresnel phase velocity of light, $W$, relative to an observer stationary in the preferred reference frame is given by equation (4).

Fresnel's reasoning must also apply to group velocity. We then would expect the magnitude of the group velocity relative to the partially dragged reference frame to be $c / n-G$ (i.e. exactly the same as it would be relative to the medium if the medium were stationary). Therefore, the Fresnel group velocity of light, $W$, relative to an observer stationary in the preferred reference frame is given by;

$$
W=c / n-G+v\left(1-1 / n^{2}\right)
$$

\section{Comparison of Equations}

In order to allow easy comparison, we summarize the equations presented above as follows;

\section{FRESNEL PHASE VELOCITY}

$$
W=c / n+v\left(1-1 / n^{2}\right)
$$

\section{EINSTEIN PHASE VELOCITY}

$$
W=c / n+v\left(1-1 / n^{2}\right)
$$

\section{FRESNEL GROUP VELOCITY}

$$
W=c / n-G+v\left(1-1 / n^{2}\right)
$$

\section{EINSTEIN GROUP VELOCITY}

$$
W=c / n-G+v\left(1-\frac{(c / n-G)^{2}}{c^{2}}\right)
$$

For phase velocity the Fresnel and Einstein equations are the same. Therefore Einstein could point to results from the Fizeau experiment with moving water as being consistent with his velocity addition formula. In his book, Pais (1982) says "He continued to say that the experimental results that had influenced him most were the observations on stellar aberration and Fizeau's measurements on the speed of light in moving water. 'They were enough', he said". But for velocities that are small compared to the velocity of light we see that phase velocity experiments do not differentiate between the Fresnel and Einstein equations. This, however is not the case for group velocity. With a little algebra Equations (10) and (11) are reduced to Equations (12) and (13) respectively for easier comparison.

\section{FRESNEL GROUP VELOCITY}

$$
W=c / n-G+v-v / n^{2}
$$

\section{EINSTEIN GROUP VELOCITY}

$$
W=c / n-G+v-v / n^{2}+\frac{v}{c}\left[\frac{2 G}{n}-\frac{G^{2}}{c}\right]
$$

Notice that the term at the end of Equation (13), which gives the difference between the Fresnel and Einstein predictions, is first order in $v / c$ and is a function of the refractive index $n$. Before proceeding to plug in real numbers for the proposed experiment a brief description of the experiment is required.

\section{Brief Description of Proposed Experiment}

Figure 1 depicts the proposed group light speed experiment, where $\mathrm{v}$ is the velocity of the experiment and observer relative to a currently unknown preferred reference frame (PRF). Light pulses from a pulsed laser are split by a fiber optic splitter as shown below. Half of a split pulse travels clockwise (left-to-right in silica fiber and then right-to-left in air-core fiber) while the other half travels counterclockwise (left-to-right in air-core fiber and then right-to-left in silica fiber). Each half of the pulse is displayed on an oscilloscope when it arrives. Although only one loop is shown, the length of the experiment can be greatly reduced by using multiple loops each with silica followed by air-core fiber. 


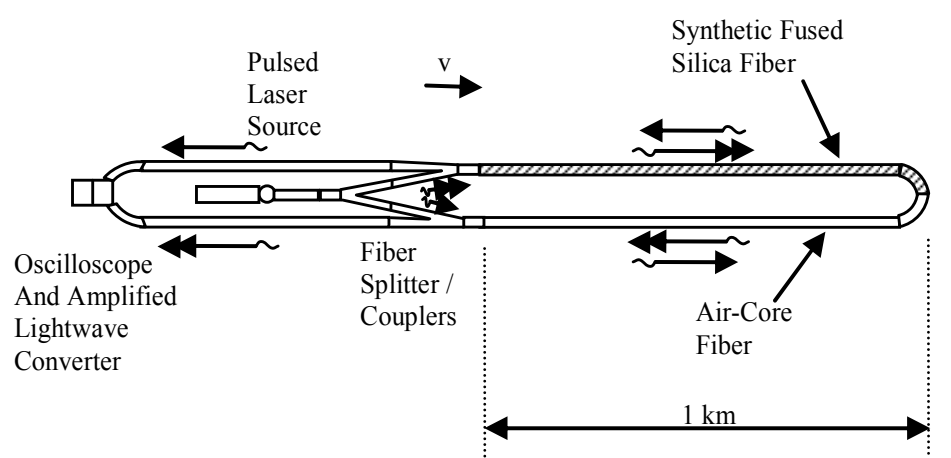

Figure 1. Proposed group light speed experiment

Our proposed experiment is a variation of an experiment performed by the Dutch astronomer Hoek (1868) and shown in Figure 2.

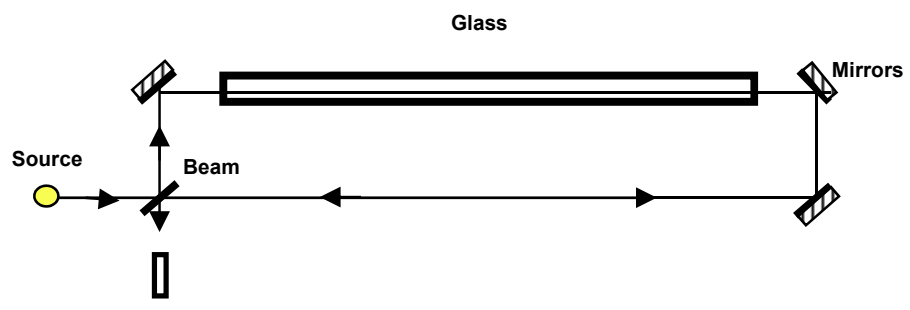

No Interference Fringe Shift Observed When Experiment Rotated

Figure 2. M. Hoek Experiment

Hoek attempted to measure an interference fringe shift following a rotation of the experiment. Our variation is that we will measure the actual arrival time difference of the light energy and require no rotation. Hoek attempted to measure a change in the difference between clockwise and counterclockwise times based on phase speed. We are attempting to measure the absolute difference in light pulse arrival times based on group speed. The Hoek experiment and similar later experiments using phase speed support both Fresnel and Einstein to the accuracy available because the predictions for phase speed differ by an extremely tiny amount.

\section{Predictions for Travel Times of the Clockwise and Counterclockwise Pulses}

When we subtract, $\mathcal{v}$, from $W$, we obtain $W_{R}$, the group speed of light relative to the moving medium as measured by the stationary observer. From Equations (12) and (13) we then have;

\section{FRESNEL GROUP VELOCITY RELATIVE TO MEDIUM}

$$
W_{R}=c / n-G-v / n^{2}
$$

\section{EINSTEIN GROUP VELOCITY RELATIVE TO MEDIUM}

$$
W_{R}=c / n-G-v / n^{2}+\frac{v}{c}\left[\frac{2 G}{n}-\frac{G^{2}}{c}\right]
$$

Please note that when $G=0$ we have the phase speed given by Pauli (1981) and Equation (14) is identical to Equation (15). 
To remain consistent with initial calculations in the earlier paper by Wagner (2008, May/June), we will assume that the top fiber shown in Figure 1 is Synthetic Fused Silica Fiber, that the bottom fiber is a vacuum, that the wavelength of the light used is $587.6 \mathrm{~nm}$ and that $v$ is parallel to the length of the experiment, as shown, with a magnitude of $365,000 \mathrm{~m} / \mathrm{s}$. Subsequent calculations with air-core for the bottom fiber predict results which are essentially the same. We have then; $v=365,000 \mathrm{~m} / \mathrm{s}$ (for all paths) For light moving left-to-right in Synthetic Fused Silica

$$
\begin{aligned}
& c=299,792,458 \mathrm{~m} / \mathrm{s} \\
& G=2,960,760 \mathrm{~m} / \mathrm{s} \\
& n=1.4585
\end{aligned}
$$

For light moving from right-to-left in Synthetic fused Silica, $w=c / n-G$ must be negative with the same magnitude so

$$
\begin{aligned}
& c=-299,792,458 \mathrm{~m} / \mathrm{s} \\
& G=-2,960,760 \mathrm{~m} / \mathrm{s} \\
& n=1.4585
\end{aligned}
$$

For light moving left-to-right in the vacuum

$$
\begin{aligned}
& c=299,792,458 \mathrm{~m} / \mathrm{s} \\
& G=0 \mathrm{~m} / \mathrm{s} \text { (i.e. group and phase speed are same) } \\
& n=1.0
\end{aligned}
$$

For light moving right-to- left in the vacuum

$$
\begin{aligned}
& c=-299,792,458 \mathrm{~m} / \mathrm{s} \\
& G=0 \mathrm{~m} / \mathrm{s} \\
& n=1.0
\end{aligned}
$$

Plugging these values into Equation (14) we have;

\section{Fresnel Group Velocity Predictions}

Light moving left-to-right in synthetic fused silica

$$
W_{R}=202,416,135 \mathrm{~m} / \mathrm{s}
$$

Light moving right-to left in synthetic fused silica

$$
W_{R}=-202,759,305 \mathrm{~m} / \mathrm{s}
$$

Light moving left-to-right in a vacuum

$$
W_{R}=299,427,458 \mathrm{~m} / \mathrm{s}
$$

Light moving right-to-left in a vacuum

$$
W_{R}=-300,157,458 \mathrm{~m} / \mathrm{s}
$$

Since the above velocities are measured in the stationary system, it is possible that the stationary observer sees a physical Lorentz contraction to the stationary length, L, of the experiment when it is moving. It is also possible that the pulse travel times measured by the stationary observer using the contracted length would then need to be converted by the pulse rate of, say, an atomic clock moving with the experiment and our observer in order to arrive at pulse travel times as measured by the moving observer. In the original paper calculations were made with and without a physical Lorentz contraction, with and without a Lorentz physical change in atomic clock rates, and with and without both. The effect on the time difference between the clockwise and counterclockwise pulse travel times was negligible. Therefore it is acceptable for our purposes, where the velocity of the medium is small compared to the velocity of light, to simply use $1000 \mathrm{~m}$ for the length of the experiment. We then have;

\section{Fresnel Laser Pulse Travel Time Predictions}

Total clockwise travel time

$$
\frac{1000 \mathrm{~m}}{202,416,135 \mathrm{~m} / \mathrm{s}}+\frac{1000 \mathrm{~m}}{300,157,458 \mathrm{~m} / \mathrm{s}}=8.2719023 \times 10^{-6} \mathrm{~s}
$$


(Please note that absolute values, i.e. magnitudes, of all right-to-left velocities are used to compute right-to-left travel times)

Total counterclockwise travel time

$$
\frac{1000 \mathrm{~m}}{299,427,458 \mathrm{~m} / \mathrm{s}}+\frac{1000 \mathrm{~m}}{202,759,305 \mathrm{~m} / \mathrm{s}}=8.2716631 \times 10^{-6} \mathrm{~s}
$$

Then the clockwise travel time minus the counterclockwise travel time equals $0.2392 \mathrm{~ns}$. This is a pulse arrival time difference which is easily measured on modern oscilloscopes.

Plugging the same values into Equation (15) we have;

\section{Einstein Group Velocity Predictions}

Light moving left-to-right in synthetic fused silica

$$
W_{R}=202,421,042 \mathrm{~m} / \mathrm{s}
$$

Light moving right-to left in synthetic fused silica

$$
W_{R}=-202,754,398 \mathrm{~m} / \mathrm{s}
$$

Light moving left-to-right in a vacuum

$$
W_{R}=299,427,458 \mathrm{~m} / \mathrm{s}
$$

Light moving right-to-left in a vacuum

$$
W_{R}=-300,157,458 \mathrm{~m} / \mathrm{s}
$$

\section{Einstein Laser Pulse Travel Time Predictions}

Total clockwise travel time

$$
\frac{1000 \mathrm{~m}}{202,421,042 \mathrm{~m} / \mathrm{s}}+\frac{1000 \mathrm{~m}}{300,157,458 \mathrm{~m} / \mathrm{s}}=8.2717825 \times 10^{-6} \mathrm{~s}
$$

Total counterclockwise travel time

$$
\frac{1000 \mathrm{~m}}{299,427,458 \mathrm{~m} / \mathrm{s}}+\frac{1000 \mathrm{~m}}{202,754,398 \mathrm{~m} / \mathrm{s}}=8.2717825 \times 10^{-6} \mathrm{~s}
$$

Then the clockwise travel time minus the counterclockwise travel time equals zero as we would expect Special Relativity Theory to Predict.

Clearly the Fresnel prediction is different than the Einstein prediction.

\section{Conclusion}

Why is the proposed experiment worth performing given all the Special Relativity experiments that support Einstein?

From the Lorentz-Fitzgerald viewpoint on the Special Relativity equations, the contraction of moving objects in the direction of their motion is considered to be physical and the change in the pulse rate of moving atomic clocks is also considered to be physical. Although this is well understood by the scientific community, it is not widely understood that the Lorentz-Poincare' viewpoint extends this physical interpretation to the synchronization of clocks at different locations in space. Poincare' showed that Lorentz's fictional "local time", introduced to show that all optical phenomena could be described in approximately the same way, could also be explained physically. If moving objects have a physical Lorentz contraction and there is a physical Lorentz change in the pulse rate of moving atomic clocks, then if two separated clocks are synchronized by a light signal whose speed is assumed (incorrectly) to be $\mathrm{c}$ the clocks will be desynchronized by the exact amount required to make all future one-way light speeds (in either direction) appear to be c. Thus in his book Galison (2003) concludes his explanation of this physical de synchronization with the statement "Two phenomena that occurred in different places could appear to be simultaneous even though they were not." This subject is addressed again and given a full mathematical foundation in a recent paper by Reignier (2004, Oct 8-9) where he says "Poincare' clarifies the physical meaning of Lorentz local time. This sentence is of uttermost importance for it defines, for the first time, the principle of synchronization of distant clocks at rest in moving bodies." 
Of the many experiments which support Special Relativity possibly only two types address the velocity addition formula. These are the Fizeau experiment with moving water and the Hoek experiment with two different mediums where the observer is stationary with respect to the experiment. Both measure changes in the phase speed of light via displaced interference fringes. None of these types of experiments have had accuracy capable of differentiating between the Fresnel drag equation and the Einstein velocity addition equation. In dealing with the Fresnel equation we have additionally considered the Lorentz-Poincare' viewpoint and found that the physical changes have a negligible effect on our prediction for velocities that are small compared to the velocity of light. Please note that the contentious physical synchronization of separated clocks is ignored because no synchronization of clocks is required in the proposed experiment.

The relativity concept which is at issue here is the most controversial concept in the Einstein viewpoint because although he assigns a one-way light speed of c, as does Poincare', he also assumes that it actually is $\mathrm{c}$ and that clocks synchronized by it are actually synchronized while Poincare' assumes that it is actually $c-v$ and that clocks are actually de synchronized by it. This is a profoundly different viewpoint because from Einstein's viewpoint we must believe that for observers moving at different speeds relative to one another, two separated events, A and B, will actually occur simultaneously for one observer but not for other observers. For the other observers, event A may actually occur before event B for one, and event B may actually occur before event A for another.

The Einstein velocity addition formula requires his viewpoint on simultaneity in order to be correct. Therefore there is every reason to perform a new direct test of this formula. The proposed experiment is a relatively cheap new direct test of Einstein's velocity addition formula. A positive result from this experiment at different orientations would lead to the identification of a local preferred reference frame where the speed of light is isotropic. This reference frame could be used in place of star reference frames for spacecraft navigation.

In addition, the acceptance of a local preferred reference frame implies the acceptance of a real physical contraction in the direction of motion relative to this reference frame and a real mass increase. In a paper by Wagner (2009, July), he explains that this means that the density of a moving mass, and therefore its refractive index and Fresnel drag will also increase. Thus a positive result from the experiment implies different physical laws in moving systems. Wagner (2009, Jan) also shows that this raises the possibility that a physical Lorentz contraction and mass increase, relative to the Fresnel partially dragged reference frame, explains the solid-state Michelson-Morley experiment null result obtained by Shamir and Fox (1969), and allows a sizeable mass to be accelerated somewhat beyond the speed of light, c, relative to the local vacuum preferred reference frame.

A positive result also brings into question accepted views on Cosmology and Quantum Theory. In the current view on cosmology, it appears that the relativity concept of space-time precludes a fixed real 3 dimensional center of the expanding universe. A positive result from the proposed experiment would allow this. The anisotropy in the cosmic microwave background radiation (CMBR) is currently interpreted to mean that we are moving at about $365,000 \mathrm{~m} / \mathrm{s}$ relative to the CMBR (sometimes considered a preferred reference frame). If, however, there is a physical center of the universe then the anisotropy might instead be caused by the fact that we are not at the physical center and that therefore the edges of the universe are at different distances from us and, based on the Hubble law, move away relative to us at different speeds (i.e. different Doppler shift). We might actually be moving much slower relative to our local preferred reference frame (referred to by relativists as the "Hubble flow" or "expansion of space") and this would explain the ongoing difficulty in detecting our motion relative to the local preferred reference frame.

In quantum theory, the current view is a probability interpretation with no real physical interpretation. If the proposed experiment produces positive results, we can consider the local preferred reference frame to have a physical existence in 3 dimensional space (perhaps based on the motion and distance of gravitational fields). This could provide the basis for development of a physical interpretation for quantum phenomena more in line with the views of two important founders of quantum theory - Einstein and Schrodinger.

The new direct test is available to us because of the development of high accuracy oscilloscopes, fiber optics, and lasers. It is the author's opinion that if Einstein were alive today he would say with his usual courage - Do this experiment.

\section{References}

Einstein, A. (1952). Relativity The Special and the General Theory, pages 39, 40. New York: Crown Publishers Inc.

Galison, P. (2003). Einstein's Clocks, Poincare's Maps, p 210. 500 Fifth Ave., New York, NY: W.W. Norton \& Company. 
Hoek, M. (1868). Détermination de la vitesse avec lacquelle est entrainée une onde lumineuse traversant un mileu en movement. Arch. Néerland., Sci. exactes naturelles, 3, 180-185.

Nascimento, U., \& de Roma, A. (1998). On the trail of Fresnel's search for an ether wind. Apeiron, 5(3-4), 181-192. Retrieved from http://redshift.vif.com/JournalFiles/Pre2001/V05NO3PDF/v05n3nas.pdf

Pais, A. (1982). Subtle is the Lord: The science and the life of Albert Einstein. page 116. Oxford University Press.

Pauli, W. (1981). Theory of Relativity, p. 17. 180 Varick Street, New York, NY: Dover Publications, Inc.

Reignier, J. (2004, Oct 8-9). Poincare' synchronization: From the local time to the Lorentz group. International Solvay Institutes for Physics and Chemistry, Proceedings of the Symposium Henri Poincare' (p. 5). Retrieved from http://www.ulb.ac.be/sciences/ptm/pmif/ProceedingsHP/Reignier.pdf

Shamir, J., \& Fox, R. (1969). A new experimental test of special relativity. Il Nuovo Cimento B Series 10, 62(2), 258-264. http://dx.doi.org/10.1007/BF02710136

Shamir, J., Fox, R., \& Lipson, S. G. (1969). A simple method of measuring very small fringe shifts. Applied optics, 8(1), 103-107. http://dx.doi.org/10.1364/AO.8.000103

Wagner, D. (2008, May/June). Fresnel Drag vs. Einstein Velocity - A Case for Further Investigation. Galilean Electrodynamics, 19(3), 43-50.

Wagner, D. (2009, Jan). Lorentz Contraction relative to Fresnel dragged reference frame explains Solid-State Michelson-Morley Experiment Null Result. Apeiron, 16 (1), 70-81. Retrieved from http://redshift.vif.com/ JournalFiles/V16NO1PDF/V16N1WAG.pdf

Wagner, D. (2009, July). Lorentz Driven Density Increase Results in Higher Refractive Index and Greater Fresnel Drag. Apeiron, 16(3), 313-322. Retrieved from http://redshift.vif.com/JournalFiles/V16NO3PDF/ V16N3WAG.pdf

Wagner, D. (2011, July 6-9). New Direct Test Proposed for Einstein's Velocity Addition Formula. 18th Annual Conference of the NPA, 8, 681-685.

\section{Copyrights}

Copyright for this article is retained by the author(s), with first publication rights granted to the journal.

This is an open-access article distributed under the terms and conditions of the Creative Commons Attribution license (http://creativecommons.org/licenses/by/3.0/). 